\title{
AN ANALOG OF THE LUSIN-PRIVALOFF RADIAL UNIQUENESS THEOREM
}

\author{
K. F. TSE
}

1. Let $D=\{|z|<1\}$ and $C=\{|z|=1\}$. For $0 \leqq \theta<2 \pi$, and $-\pi / 2<\phi<\pi / 2$, let $\rho(\theta)=\left\{r e^{i \theta}: 0 \leqq r<1\right\}$ and $\rho(\theta, \phi)=\left\{e^{i \theta}\left(1-r e^{i \phi}\right)\right.$ $: 0<r \leqq \cos \phi\}$. If $f(z)$ is a meromorphic function in $D$, and $G \subseteq D$ such that $e^{i \theta} \in \bar{G} \cap C$, then $C_{G}\left(f, e^{i \theta}\right)$ represents the cluster set of $f(z)$ at $e^{i \theta}$ restricted in $G$. $F(f)$ will denote the set of Fatou points of $f(z)$ on $C$. (For a definition of Fatou points, see [6, p. 61].) Finally, a subset $S$ of $C$ is said to be metrically dense on an arc $A$ of $C$ if meas $\left(A^{\prime} \cap S\right)>0$ for each subarc $A^{\prime}$ of $A$.

Barth and Schneider have proved the following analog of the F. and M. Riesz uniqueness theorem for bounded holomorphic functions.

Theorem A [3, Theorem 1]. Let $\mu$ be a decreasing function on $[0,1)$ such that $\lim _{r \rightarrow 1} \mu(r)=0$, and let $S$ be a subset of $C$ of second category. If $f(z)$ is a bounded holomorphic function in $D$ and $\left|f\left(r e^{i \theta}\right)\right|$ $=o(\mu(r))$ for each $e^{i \theta}$ in $S$, then $f(z) \equiv 0$.

In Remark 4 of [3], the question whether the above theorem holds for holomorphic functions has been raised. We shall show that it even holds for meromorphic functions.

TheOREM. Let $\mu$ and $S$ be the same as stated in Theorem A. If $f(z)$ is a meromorphic function in $D$ and $\left|f\left(r e^{i \theta}\right)\right|=o(\mu(r))$ for each $e^{i \theta}$ in $S$, then $f(z) \equiv 0$.

Before showing the proof, we wish to remark that our theorem is an analog of the Lusin-Privaloff uniqueness theorem which states:

If $f(z)$ is a holomorphic function in $D$ and $\lim _{r \rightarrow 1} f\left(r e^{i \theta}\right)=0$ for each $e^{i \theta}$ in $S, S$ being both metrically dense and of second category on an arc $A$ of $C$, then $f(z) \equiv 0$.

2. To prove our theorem, we need two lemmas. The first one is very elementary in applying the notion of Baire categories. The second one is an extension of [4, Lemma 1$]$.

Lemma. 1. Let $S$ be a set of second category in an interval $J$, then there exists a subinterval $I$ of $J$ such that $S \cap I$ is both dense and of second category in $I$.

Received by the editors May 17, 1969 and, in revised form, June 28, 1969. 
Proof. The existence of an interval in which $S$ is of second category and of an interval in which $S$ is dense are immediate consequences of the definition of "second category." We must prove the existence of an interval with both of these properties. By the remark following the proof of $[5$, Theorem 35, p. 201], there exists a nonempty closed subinterval $I^{\prime}$ of $J$ such that the intersection of $S$ with any neighborhood $N$ of a point in $I^{\prime}$ is of second category in $I^{\prime}$. Thus a subinterval $I$ of $I^{\prime}$ in which $S$ is dense has all the properties required.

Applying Lemma 1, it is easy to see that we can modify the proof of $[4$, Lemma 1, pp. 170-171] and obtain

Lemma 2. Suppose that $f(z)$ is a meromorphic function in $D$ and that for some fixed $\phi,-\pi / 2<\phi<\pi / 2$, and some complex number $\beta$, finite or infinite, there exists a set $S$ of second category on $C$ and such that

$$
\beta \notin \bigcup_{e^{i \theta} \in S} C_{\rho(\theta, \phi)}\left(f, e^{i \theta}\right) .
$$

Then there exists an arc $A$ on $C$ such that

(1) Either $f(z)$ or $1 /(f(z)-\beta)$ is uniformly bounded in a relaive neighborhood of $A$ in $D$ according as $\beta=\infty$ or $\beta \neq \infty$,

(2) $A \cap S$ is dense in $A$, and

(3) $A \cap S$ is of second category in $A$.

3. We now proceed to prove our theorem. Since $\lim _{r \rightarrow 1} \mu(r)=0$ and $\left|f\left(r e^{i \theta}\right)\right|=o(\mu(r))$ for each $e^{i \theta}$ in $S$, we have

$$
\infty \notin \bigcup_{e^{i \theta} \in S} C_{\rho(\theta)}\left(f, e^{i \theta}\right) .
$$

Note that $S$ is of second category. By Lemma 2, there exists an arc' $A$ of $C$ such that $f(z)$ is uniformly bounded in a relative neighborhood of $A$ in $D$ and that $A \cap S$ is of second category in $A$.

On the other hand, by a theorem [2, p. 6] of Barth and Schneider, there exists a nonconstant holomorphic function $g(z)$ in $D$ such that

$$
\max _{0 \leqq \theta<2 \pi}\left|g\left(r e^{i \theta}\right)\right|<1 / \mu(r) \text { and } \lim _{r \rightarrow 1} g\left(r e^{i \theta}\right)=0
$$

for each $e^{i \theta}$ in $T$, where $T$ is a subset of $C$ of measure $2 \pi$. Now, consider the function $h(z)=f(z) g(z)$. Note that

$$
\lim _{r \rightarrow 1} h\left(r e^{i \theta}\right)=0
$$

for each $e^{i \theta}$ in $(S \cup T) \cap A$. Since $(S \cup T) \cap A$ is both metrically dense and of second category in $A, h(z)$ satisfies the hypotheses of [4, Theorem 1] and we have $h(z) \equiv 0$. Hence $f(z) \equiv 0$. 
4. Gauthier (in a written communication to the author) has kindly pointed out that our theorem is sharp in the following sense:

TheOREM B. Let $\mu$ be as in Theorem A and $S$ be any set of first category on $C$. Then there exists a nonconstant holomorphic function $f(z)$ in $D$ such that $\left|f\left(r e^{i \theta}\right)\right|=o(\mu(r))$ for each $e^{i \theta}$ in $S$.

Proof. Since $S$ is of first category, $S=\cup_{n=1}^{\infty} S_{n}$, where $S_{n}$, for each $n=1,2, \cdots$, is nowhere dense on $C$. Thus $S^{\prime}=\bigcup_{n=1}^{\infty} \bar{S}_{n}$ is also of first category on $C$ and $S \subseteq S^{\prime}$. Consider the set

$$
E^{\prime}=\bigcup_{n=1}^{\infty}\left\{r e^{i \theta}: e^{i \theta} \in \bar{S}_{n}, 1-1 / 2 n \leqq r<1\right\} .
$$

Choose $\theta_{0}$ such that $e^{i \theta_{0}} \notin S^{\prime}$ and let $E=E^{\prime} \cup \rho\left(\theta_{0}\right)$. Note that $E$ is relatively closed and nowhere dense in $D$. We define

$$
\begin{aligned}
\phi(z) & =0, & & z \in E^{\prime}, \\
& =1, & & z \in \rho\left(\theta_{0}\right) .
\end{aligned}
$$

$\phi(z)$ is continuous on $E$. Hence by a theorem of Arakeljan (see Arakeljan [1]), there exists a holomorphic function $f(z)$ in $D$ such that $|f(z)-\phi(z)| \leqq \mu(|z|)(1-|z|)$ for each $z$ in $E$. It follows that $\left|f\left(r e^{i \theta}\right)\right|$ $=o(\mu(r))$ for each $e^{i \theta}$ in $S^{\prime}$ (note that $S \subseteq S^{\prime}$ ), and $f(z)$ ₹ 0 because $f(z)$ is near to one on $\rho\left(\theta_{0}\right)$.

5. The author wishes to thank Dr. P. Gauthier for his kind permission to include Theorem B in this paper and Mr. P. S. Pun for a stimulating discussion.

\section{REFERENCES}

1. N. U. Arakeljan, Uniform and tangential approximation with analytic functions, Izv. Akad. Nauk Armjan. SSR Ser. Mat. 3 (1968), no. 45, 273-286. (Russian)

2. K. F. Barth and W. J. Schneider, On the impossibility for extending the Riesz uniqueness theorem to functions of slow growth, Ann. Acad. Sci. Fenn. Ser. AI 432 (1968), 9 pp.

3. - An asymptotic analog of the $F$. and M. Riesz radial uniqueness theorem, Proc. Amer. Math. Soc. 22 (1969), 53-54.

4. E. F. Collingwood, On the linear and angular cluster sets of functions meromorphic in the unit circle, Acta Math. 91 (1954), 165-185. MR 16, 460. 1136.

5. J. L. Kelley, General topology, Van Nostrand, Princeton, N. J., 1955. MR 16,

6. K. Noshiro, Cluster sets, Ergebnisse der Mathematik und ihrer Grenzgebiete, Heft 28, Springer-Verlag, Berlin and New York, 1960. MR 24 \#A3295.

Syracuse University, Syracuse, New York 13210 